\section{Randomization tests: Viable alternatives to normal curve tests}

\section{R. B. MAY, M. E. J. MASSON, and M. A. HUNTER University of Victoria Victoria, British Columbia, Canada}

Fisher (1935) proposed that randomization tests of scores are appropriate for analysis of research involving random allocation of experimental units to conditions. Pitman (1937) provided a theoretical framework for these tests, and others (e.g., Feinstein, 1973; Kempthorne, 1955,1979 ) found the tests conceptually superior to normal curve tests such as $t$ and $F$.

Because of computational problems, however, randomization tests are rarely used by researchers. Instead, in situations in which scores would most appropriately be analyzed by randomization tests, researchers either diminish their data and use computationally easier rank tests, or continue to use parametric tests because of tradition or because parametric tests often give approximately the same probability value as randomization tests of scores (see Boik, 1987; Bradbury, 1987; Collier \& Baker, 1966; McHugh, 1963). Unfortunately, rank tests are less powerful than randomization tests of scores, and the results of parametric tests and randomization tests do not always agree (Edgington, 1987).

Randomization tests are computed in the following manner: A test statistic such as the difference between two means is computed for the data; then the data are permuted repeatedly and the statistic is computed for each permutation. The proportion of data permutations with test-statistic values greater than or equal to the obtained result is the probability (or significance) value.

A major limitation of randomization tests of scores is that the number of permutations required to produce an exact probability increases dramatically as the numbers of conditions and units per condition increase. For example, with two independent groups of four cases, there are 70 possible permutations of the obtained data, but with four groups of four cases, there are over 63 million possible permutations. A computer-intensive approach makes it possible to compute probability values with the randomization model and relatively little computing time. Edgington (1987) provided FORTRAN programs that may be used to compute these tests in several situations. His programs have been adapted into a single program, NPSTAT, designed primarily as a pedagogical tool illustrating the nature of randomization tests of scores and contrasting them with normal curve tests. Possible pedagogical ad-

\footnotetext{
Correspondence may be addressed to Richard B. May, Department of Psychology, University of Victoria, P.O. Box 1700, Victoria, British Columbia V8W 2Y2, Canada.
}

vantages of teaching these tests have been discussed by May and Hunter (1988).

The program. NPSTAT compares randomization results for scores and for ranks (e.g., Mann-Whitney $U$ ) with normal curve results for several research contexts. Scores and ranks may be analyzed for either betweengroup or within-subject one-factor designs with two to eight groups. With between-group designs, groups may be equal or unequal in size. Simple correlations may be computed for two variables at a time. Frequency data are treated for the one-sample case (e.g., binomial test) and the $2 \times 2$ case yielding the Pearson chi-square, Yates correction, and an exact probability from the randomization test ("Fisher's exact test').

Exact versus estimated probabilities. Except in the case of trivially small problems, the number of possible permutations may be many thousands or millions, and a choice must be made between using all possible permutations or a randomly selected subset. If all possible values are computed, the resulting probability will be an exact value, but it may take many minutes to obtain. The alternative is to chose some number, such as 2,000 randomly selected permutations. This option results in an estimate of the exact value and requires much less computation time (e.g., 10 to $20 \mathrm{sec}$ on an 80286 processor with math coprocessor). Both options are available on NPSTAT for each type of analysis (independent groups, dependent groups, correlation). The program also provides a listing of the null frequency distribution based on all permutations used in the analysis. This reference set gives the frequency of occurrence of each possible value of the statistic computed (e.g., mean1-mean2) along with its individual and cumulative probabilities.

Computer and language. Our adaptation of Edgington's programs is written in C for the IBM PC XT, AT, $\mathrm{PS} / 2$, or compatibles under MS-DOS/PC-DOS 2.0 or higher. NPSTAT will use a math coprocessor if one is available.

Core requirement. A minimum of $430 \mathrm{~K}$ of free RAM is needed.

Input. NPSTAT is menu driven, and there is a help/ information library to assist with choices on each menu. Data files may be stored, retrieved, and edited. Files generated by other programs may be imported if they are in the correct ASCII format. A help menu gives access to detailed instructions for saving and importing files.

The program may be customized for black and white or color monitors, IBM or other printers, and so forth. A help system gives access to detailed instructions for customization.

Output. Descriptive statistics, parametric analysis (e.g., $t$ or $F$ ), and randomization analysis are given for each analysis of scores. Options for viewing and printing include raw data, statistics, and a listing of the null fre- 
quency distribution of permutation outcomes (unique to each set of scores).

Limitations. The program does not treat factorial designs or multiple dependent variables. There can be no more than 2,000 distinct outcomes (values of the test statistic) for the randomization tests. If this limit is exceeded, the randomization analysis terminates after 2,000 distinct outcomes have been produced. The reported probability will be based on the number of permutations required to reach 2,000 distinct outcomes.

For analysis of independent samples of unequal size, the smallest sample should be entered first and the largest sample entered last. Computation time is much longer if the opposite order of data entry is used.

Availability. A copy of the program may be obtained from the authors for an $\$ 8$ handling fee (or $\$ 6$ if a formatted diskette is included with the request). Requests should specify whether a 5.25 - or a 3.5 -in. diskette is preferrred.

\section{REFERENCES}

BoIk, R. J. (1987). The Fisher-Pitman permutation test: A non-robust alternative to the normal theory $F$ when variances are heterogeneous. British Joumal of Mathematical \& Statistical Psychology, 40, 26-42.
BRadBURY, I. (1987). Analysis of variance versus randomization tests-a comparison. British Journal of Mathematical \& Statistical Psychology, 40, 177-187.

Collier, R. O., JR., \& Baker, F. B. (1966). Some Monte Carlo results on the power of the $F$ test under permutation in the simple randomized block design. Biometrika, 53, 199-203.

EdGington, E. S. (1987). Randomization tests (2nd ed.). New York: Marcell Dekker.

Feinstein, A. R. (1973). Clinical biostatistics XXIII: The role of randomization in sampling, testing, allocation, and credulous idolatry (Part 2). Clinical Pharmacology \& Therapeutics, 114, 898-915.

Fisher, R. A. (1935). The design and analysis of experiments. Edinburgh: Oliver \& Boyd.

KEMPTHORNE, O. (1955). The randomization theory of experimental inference. Joumal of the American Statistical Association, 50, 946-967.

KEMPTHORNE, O. (1979). Sampling inference, experimental inference, and observation inference. Sankhya: The Indian Joumal of Statistics, 40, 115-145.

MAY, R. B., \& Hunter, M. A. (1988). Interpreting students' interpretation of research. Teaching of Psychology, 15, 156-158.

McHugh, R. B. (1963). Comment on "'Scales and statistics: Parametric and nonparametric." Psychological Bulletin, 60, 350-355.

Pitman, E. J. G. (1937). Significance tests which may be applied to samples from any populations. Journal of the Royal Statistical Society, 4, 119-130.

(Manuscript received September 12, 1988; revision accepted for publication June $1,1989$. 